\title{
Real-Time Algorithm For Adaptive Beamforming Using Cyclic Signals '
}

\author{
Shiann-Jeng $\mathrm{Yu}^{*}$ and Ju-Hong Lee \\ Department of Electrical Engineering \\ National Taiwan University \\ Taipei, Taiwan, R.O.C.
}

\begin{abstract}
Adaptive beamforming using signal cyclostationarity can preserve the desired signal and cancel the interferers without prior information of the steering vector. In this paper, we consider the Cross-SCORE processor which is one of this class of beamformers and uses time-consuming eigen value decomposition (EVD) to compute weight vectors. Thus, this processor is not suitable for real-time processing. We here apply modular Gram-Schmidt Orthogonalization (GSO) structure in conjunction with power normalization scheme to the Cross-SCORE processor and propose a LMS based adaptive algorithm to update weight vectors. Due to the pipeline and parallel properties of the modular GSO structure, our approach is very suitable for real-time processing and the required computing time for the array to process an output is $\mathrm{O}(N)$, where $N$ is the number of array elements.
\end{abstract}

\section{Problem Formulation}

Consider a narrowband array with $N$ isotropic receiving sensor elements. Let the signal data vector $X(l)$ received by the sensors and $I^{\prime}(l)=X^{*}(l+\tau) \exp (j 2 \pi(r l)$, where $\tau$ is a time delay and the cyclic frequency $\alpha$ is equal to doubled symbol rate or multiple baud rates or both. The CrossSCORE processor computes the control vector $C$ and weight vector $W$ by solving the following problem

$$
\text { Maximize } \rho=\frac{\mid W^{\prime \prime} R_{x u t}\left(\left.!\right|^{2}\right.}{\left(W^{\prime \prime} R_{x x} W\right)\left(c^{\prime \prime} R_{n u}(!)\right.} \text { subject to } W^{\prime \prime} W=C^{\prime \prime} C^{\prime}=1
$$

In (1), $R_{x x}=\left\langle X(t) X^{\prime \prime}(t)>_{\infty}, R_{x u}=\left\langle X(t) U^{H}(l)>_{\infty}\right.\right.$, and $R_{u u}=\left\langle U(t) U^{H}(t)\right\rangle_{\infty}$, where $\langle.\rangle_{\infty}$ denotes infinite time average. Thus, the optimal weight vectors for $W$ and $C$ can be found as the eigenvectors with the largest eigenvalues of

$$
R_{x x}^{-1} R_{x u} R_{u u}^{-1} R_{u x} \text { and } R_{u u}^{-1} R_{u x} R_{x x}^{-1} R_{x u}
$$

respectively. Using the EVD, the Cross-SCORE requires $O\left(N^{3}\right)$ complex multiplications.

'This work was supported by the National Science Council Under Grant NSC84-2213-E002-07! 


\section{The Proposed Technique}

In this paper, we notice that finding the eigenvectors of (2) is equivalent to finding the eigenvectors of

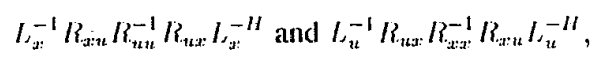

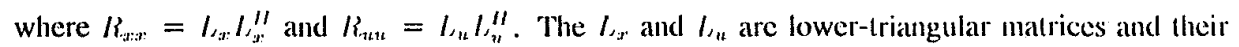
inverses exist. Therefore, we can express the optimal weight vectors as $W=L_{x}^{-1} W_{L}$ and $C^{\prime}=$ $L_{u}^{-H} C_{L}$ with $W_{L}$ and $C_{L}$ being the largest left and right singular vectors of $R_{L x u}=L_{x}^{-1} R_{x u} L_{u}^{-11}$, respectively.

There exists a number of methods to factorize $R_{x x}=R_{u t u}^{*}$. We here employ the modular GSO structure. Let the equivalent transformation matrix of the GSO be expressed as a upper triangular matrix $V_{x}$. Forming the correlation matrix $P_{x}^{2}=\operatorname{diag}\left\{P_{x 1}^{2}, P_{x 2}^{2}, \cdots, P_{x N}^{2}\right\}=V_{x}^{H} R_{x x} V_{x}$, we can find $L_{x}^{-H}=V_{x} I_{x}^{-1}$. In practice, $V_{x}$ and $L_{x:}$ are updated recursively, we may rewrite $L_{x}^{-H}(l)=$ $V_{x}(t) P_{x}^{-1}(t)$.

Given $L_{x}$ and $L_{u}$, we rewrite the cost of (1) as

$$
\rho=\frac{\left|W_{L}^{H} R_{L x u} C_{L}\right|^{2}}{W_{L}^{H} W_{L} C_{L}^{H} C_{L}} .
$$

If we restrict that the weight vector $W_{L}$ and $C_{L}$ are unit-norm, $\rho=\left|W_{L}^{H} R_{L x u} C_{L}\right|^{2}$ becomes a simple quadratic cost function. Therefore, using the instantaneous gradicnt, we can develop the LMS based algorithm as follows.

$$
\begin{array}{ll}
W_{L}(t)=W_{L}(t-1)+\mu_{w} X_{L}(t) U_{L}^{H}(t) C_{L}(t-1), & W_{L}(t) \leftarrow W_{L}(t) /\left\|W_{L}(t)\right\|, \\
C_{L}(l)=C_{L}(l-1)+\mu_{c} U_{L}(l) X_{L}^{\prime \prime}(l) W_{L}(l-1), & C_{L}(l) \leftarrow C_{L}(l) /\left\|C_{L}(l)\right\|, \\
W(l)=\tau_{u} L_{x}^{-\prime \prime}(l) W_{L}(l), & C_{(}(l)=\tau_{c} L_{u}^{-\prime \prime}(l) C_{L}(l),
\end{array}
$$

where $X_{L}(t)=V_{x}^{H}(t) X(t), U_{L}(t)=X_{L}^{*}(t+\tau) \mathrm{e}^{j 2 \pi \alpha t}, \mu_{w}$ and $\mu_{c}$ are step sizes, and $\tau_{w}$ and $\tau_{c}$ are two scalars. The configuration of the proposed approach is shown in Figure 1 .

We have noticed that the proposed algorithm is affected by the initial guesses of the weight vectors $W_{L}$ and $C_{L}$. For some applications, we may roughly estimate the direction of the desired signal by using the beamspa?e preprocessor and then use the estimated directional steering vector as the initial guess. If we pick the initial weights $W_{L}(0)$ and $C_{L}(0)$ such that $L_{x}^{-H}(0) W_{L}(0)=$ $\gamma_{w} \hat{S}_{d l}$ and $L_{u}^{-H}(0) C_{L}(0)=\gamma_{c} \hat{S}_{d}^{*}$, where $\hat{S}_{d}$ is the initial estimate of the desired signal direction, $\gamma_{w}$ and $\gamma_{c}$ are constants such that $W_{L}(0)$ and $C_{L}(0)$ are unit-norm.

\section{Simulation Examples}

We here provide an example to show the performance of the proposed algorithm. A uniform linear array with $N=6$ and half-wavelength element spacing is employed. The scenario of 
the signal sources is shown in Table 1 . The initial $L_{x}(0)=I$ and $R_{i i}(0)=10^{-6}$ for $i=1$, $2, \cdots, 6$. The cyclic frequency $r=0.2$. We cloose $\mu_{w}=0.05 /\left(X_{l}^{\prime \prime}(1) X_{L}(t)\right)$ and $\mu_{i}=$ $0.05 /\left(1 l_{l}^{\prime \prime}(l) U_{l}(l)\right)$. The initials $W_{l},(0)$ and $C_{l}^{\prime}(0)$ are the nomalized Hamming window functions with maximum gain in the broadside. Figure 2 presents the output signal-to-inter ference plus noise ratio (SINR) versus number of snapslots. The results alre averaged by 100 independent runs. Fig. 2(a) uses $\tau=0$ and Fig. 2(b) uses $\tau=\Delta{ }^{\prime}$, where $\Delta T^{\prime}=1$ is the sampling interval. They show that the Cross-SCORE [2] has the fastest convergence speed at the expense of large computation load. However, the proposed algorithm can be implemented in real time and achieve better performance when it converges for different values of $\tau$.

\section{References}

[1] W. A. Gardner, STATISTICAL SPECTRAL ANALYSIS. Prentice-Hall, Englewood Cliffs, New Jersey, 1988.

[2] B. G. Agec, S. V. Schell, W. A. Gardner, "Spectral self-coherence restoral: a new approach to blind adaptive signal extraction using antenna arrays," Proc. IEEE, Vol. 78, pp. 753-760, Apr. 1990.

Table 1 The scenario of Figure 2

\begin{tabular}{|l|l|l|c|r|l|}
\hline Signal & SNR (dB) & Symbol Rate & Baud Rate & DOA & Pulse Shape \\
\hline BPSK $^{*}$ & 3 & 0.1 & $1 / 3$ & $0^{\circ}$ & cosine \\
\hline BPSK & 20 & 0.35 & $1 / 5$ & $35^{\circ}$ & cosine \\
\hline QAM & 20 & 0.15 & $1 / 7$ & $20^{\circ}$ & \\
\hline QAM & 20 & 0.25 & $1 / 8$ & $-25^{\circ}$ & \\
\hline
\end{tabular}

* is the desired signal

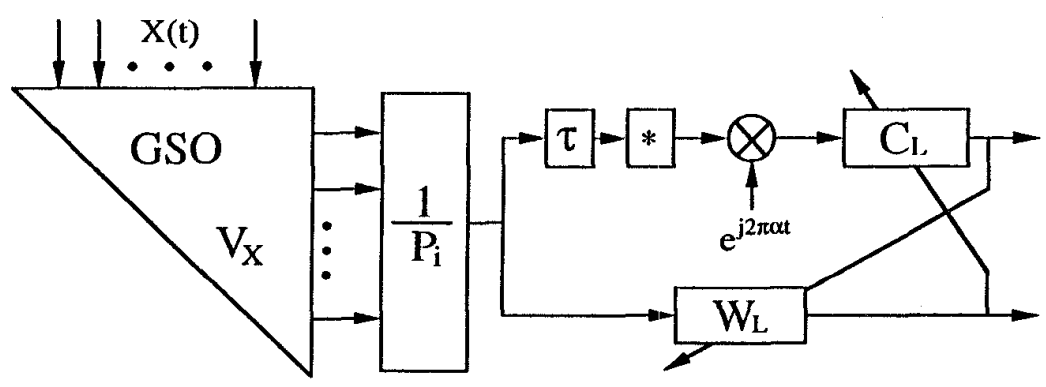

Figure 1. The Proposed Configuration 


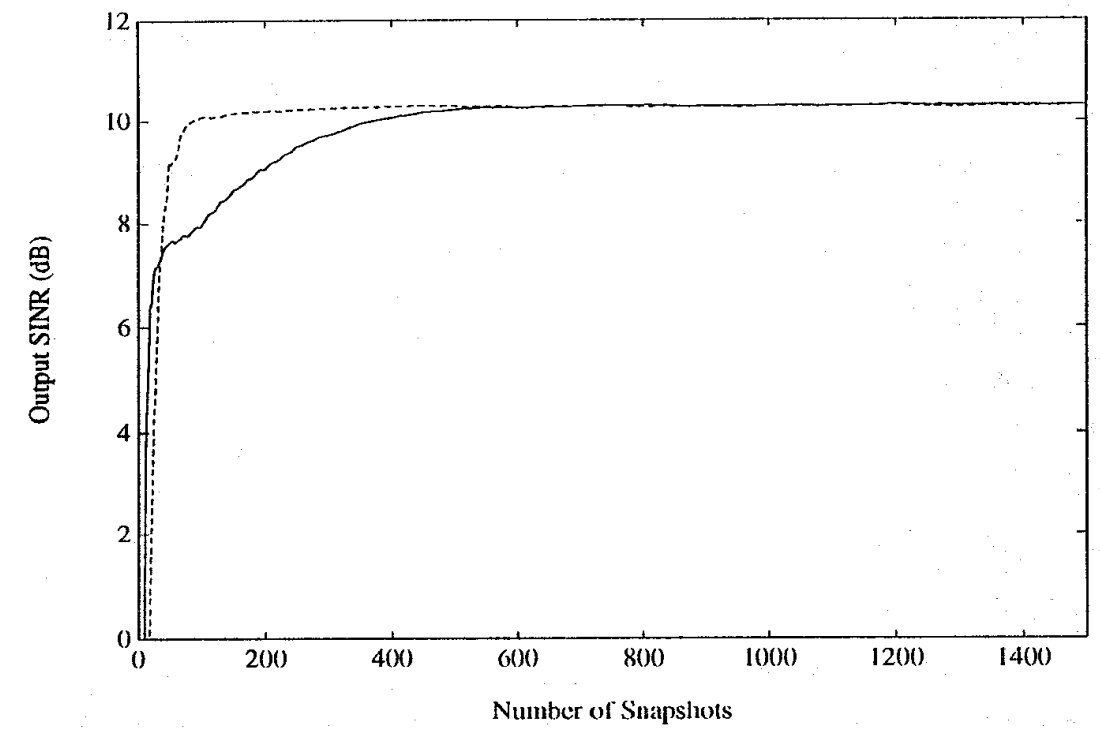

Fig. 2(a) The Output SINR versus Number of Snapshots. Solid line: The Proposed Approach. Dash line: The EVD based Cross-SCORE. $\tau=0$.

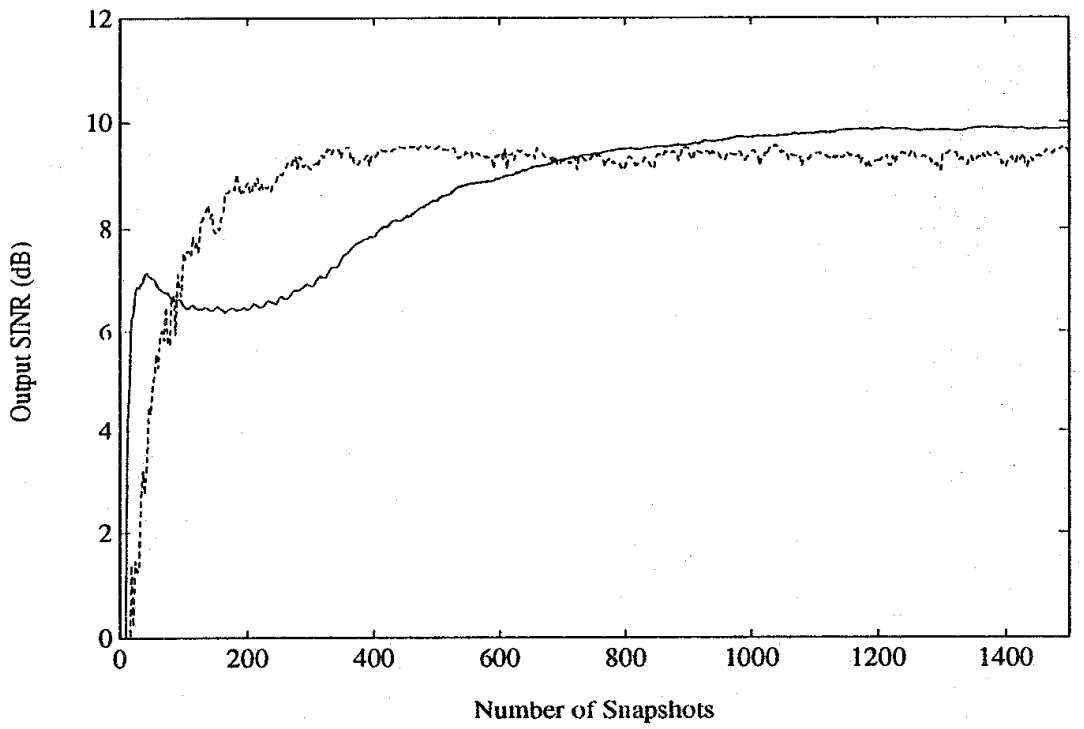

Fig. 2(b) The Output SINR versus Number of Snapshots. Solid line: The Proposed Approach. Dash line: The EVD based Cross-SCORE. $\tau=\Delta T$. 